\title{
CASOS DE ENFERMEDAD DE CHAGAS DIAGNOSTICADOS EN LIMA*
}

Alberto Cornejo Donayre. Alfredo Berrocal. Edgardo Cubas N.

Hace cuatro años que uno de nosotros tuvo ocasión de hacer una revisión completa sobre la presencia de la enfermedad de Chagas en el Perú (1), con motivo de los Sexios Congresos Internacionales de Medicina Tropical y Paludismo realizados en Lisboa en Setiembre de 1958. En esa oportunidad señalamos, que el mayor número de investigaciociones efectuadas en los últimos 10 años, había demostrado que ésta enfermedad tenía mucho mayor diseminación de la que inicialmente se había supuesto y que su área de dispersión se estaba incrementando constantemente, debido a múltiples factores epidemiológicos, lo cual constituía un problema importarite de Salud Pública, ya que se había demostrado la presencia de casos, del agente etiológico, del reservorio - de triatóminos en 15 departamentos del Perú, debiendo destacarse que en la mayoría de los restantes no se había efectuado investigaciones en relación con esta entermedad.

A fines de 1957 comprobamos la presencia de Triatoma infestans en Lima, en una casa residencial del distrito de Pueblo Libre, donde posiblemente habia ingresado procedente de la casa colindante (1), en la que residia una familia que frecuentemente recibia encomiendas procedentes de Arequipa y Tacna, que son departamentos intensamente infestados por T. infestans. El año siguiente se publicó el interesante trabajo de A. Tejada y B. Llanos (2), quienes demostraron la infestación de 10 viviendas situadas dentro de la zona urbana del distrito de la Victoria, que es la zona más poblada de Lima, y señalaron el hallazgo de insectos adultos, nintas, larvas y huevos de T. infestans. Felizmente

(*) La primera parte de este trabajo efectuodo en la Cótedro de Medicino Tropical de la Facultod de Medicina de Son Fernondo, fue publicoda en Amer. J. Trop. Med., 11, 610, 1962. 
que ninguno de los especímenes capturados en las oportunidades mencionadas, estaba infectado por T. cruzi.

Los hallazgos mencionados y el considerable incremento en la población de Lima durante los últimos 21 años, debido principalmente, a la afluencia de personas procedentes de todas las regiones del territorio nacional en busca de mejores condiciones de vida y mayores posibilidades de trabajo, nos indujo a tratar de determinar la presencia ile casos de enfermedad de Chagas en residentes de Lima, que habían nacido o residido por varios años en las zonas donde ésia enfermedad es endémica.

La población de Lima se ha incrementado de 520,528 según el censo de 1940, a 1'729,982 habilantes, de acuerdo a los datos oficiales del censo efectuado el 2 de Julio de 1961. Esto representa el $232.4 \%$ de aumento, correspondiendo las mayores cifras a pobladores procedentes de otros departamentos, que se han establecido en las zonas urbanas de la ciudad o que han formado las nuevas barriadas que circundan la capital. En éstas últimas, hay condiciones sanitarias muy deficientes, se carece de servicios de agua y desagüe, la vivienda es rústica, sobrepoblada y tiene condiciones muy favorables para la diseminación de la Trypanosomiasis Americana, en la que el hombre tiene rol tan importante.

\section{MATERIAL Y METODOS}

Durante los últimos 30 meses hemos estudiado 214 personas que tenían varios años de residencia en Lima. Entre ellos hubo 200 hombres y 14 mujeres. Todos fueron seleccionados sobre la base de su ro sidencia anterior en zonas chagásicas y algunos fueron remitidos a nuestro laboratorio por médicos cardiólogos, porque además de sus ontecedentes epidemiológicos presentaban síntomas y signos de afección cardiovascular. El de menos edad 9 años y el de maryor 74. En cada uno había antecedenies de contactos con triatóminos y no pocos informaron haber sido picados por estos insectos.

Las 214 personas consideradas en este trabajo han sido distribuídas en grupos de edades: de 9 a 19 años, hubo 145; de 20 a 40 años, 30; de 41 a 60 años, 32; y de 60 y más años, 7. Cuadro $N^{\circ}$ l.

La residencia previa de todos ellos fue en los 10 departamentos que a continuación se indica: 4 habían nacido o residido en Tumbes; 70 en Piura; 12 en San Martín; 6 en Amazonas; 34 en Cajamarca; 1 en 
Lambayeque; $5 \mathrm{l}$ en Arequipa; 15 en Moquegua; 20 en Tacna y 1 en Loreto. Cuadro $\mathrm{N}^{\circ} 1$.

En los 214 se practicó la reacción de fijación del complemento de Machado Guerreiro y solamente en los que ésta resultó positiva y en los familiares de ellos, se aplicó el métódo de xenodiagnóstico. Además, en reducido número se investigó la prasencia de T. cruzi en frotıs y gota gruesa de sangre periférica.

Cuadro No 1. Distribución por grupos de edad, sexo y lugar de nacimiento o residencio anferior de 214 residentes de Lima

\begin{tabular}{|c|c|c|c|c|c|c|c|c|c|c|c|c|c|}
\hline $\begin{array}{l}\text { Grupos } \\
\text { de } \\
\text { edad }\end{array}$ & Sexo & 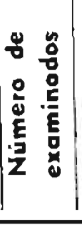 & E & $\frac{0}{3}$ & 1 & 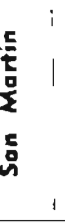 & $\begin{array}{l} \\
0 \\
0 \\
0 \\
0 \\
8 \\
8\end{array}$ & 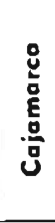 & 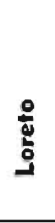 & 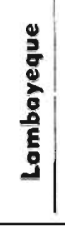 & 递 & 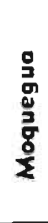 & : \\
\hline $\begin{array}{l}9-19 \\
\text { oños }\end{array}$ & $\begin{array}{l}M \\
F\end{array}$ & $\begin{array}{r}144 \\
1\end{array}$ & 4 & 61 & 1 & 2 & 5 & 24 & & & $\begin{array}{r}26 \\
1\end{array}$ & 3 & 9 \\
\hline \multirow[t]{2}{*}{$20-40$} & $M$ & 23 & & 4 & & & 1 & 2 & & & 10 & 2 & 4 \\
\hline & $F$ & 7 & & 2 & & & & & & 1 & 2 & & 2 \\
\hline \multirow[t]{2}{*}{$41-60$} & $M$ & 27 & & 2 & & & & 7 & 1 & & 10 & 6 & 1 \\
\hline & $\mathrm{F}$ & 5 & & & & & & & & & 1 & 2 & 2 \\
\hline $61 \mathrm{y}$ & $M$ & 5 & & 1 & & & & 1 & & & 1 & & 2 \\
\hline más & $\mathrm{F}$ & 2 & & & & & & & & & & 2 & \\
\hline
\end{tabular}

En la prueba de fijación del complemento de Machado-Guerreiro, se empleó la técnica usada en al Departamento de Parasitología de la Universidad de Chile, es decir con el método del $50 \%$ de hemólisis de Bozicevich y colaboradores (3), aplicada al diagnóstico de la enfermedad de Chagas (4). Inicialmente utilizamos antígeno de $T$. cruzi que gentilmente nos proporcionó el Profesor Amador Neghme y posteriormente, antígeno preparado por nosotros, siguiendo la técnica de la Universidad de Chile (4), con una mezcla de 7 cepas peruanas de T. cruzi, que hemos aislado de pacientes y de $\mathrm{T}$. infestans.

Con la finalidad de chequear nuestro trabajo, remitimos al Instituto Oswaldo Cruz once muestras de suero correspondientes a igual número de pacientes del grupo estudiado y en las que se incluía muestras positivas y negativas. El Dr. Julio Muñiz, Jefe de la División para Endemias en el referido Instituto, informó resultados iguales a los obtenidos por nosotros. 
Para la prueba de xenodiagnóstico se utilizó ninfas de Triatoma infestans nacidas en el criadero de nuestro laboratorio y que habian sido alimentadas exclusivamente con sagre de gallina. En cada xenodiagnóstico se empleó 10 ninfas, asegurándonos que todas hubiesen succionado sangre después de realizada la prueba y se les sometló $a$. dos exámenes de heces; el primero 10 días después de realizada la prueba; y el segundo a los treinta días. Sesenta días después, todos los insectos fueron disectados, examinándose cuidadosamente el intestino medio y el posterior en busca de $\mathrm{T}$. cruzi.

Cuadro No 2. Enfermedad de Chagas en 214 Residentes de Lima. Señalándose lugar de nacimiento o residencia anterior

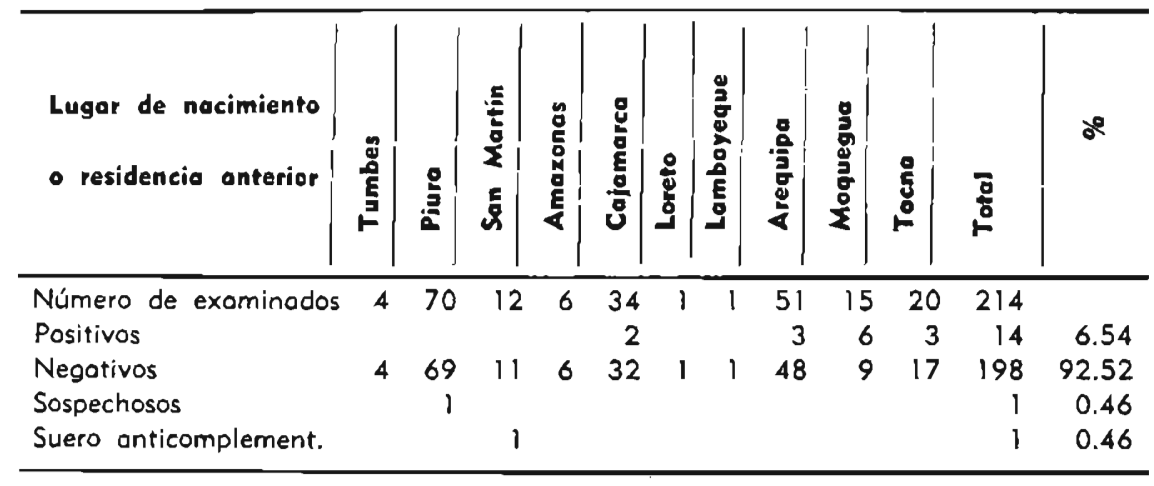

En los casos de xenodiagnóstico positivo se aisló las nuevas cepas en ratas y en medios de cultivo.

En 2 pacientes con parasitemia, se les administró Primaquina, a la dosis de 15 mgr. diorios durante 14 días consecutivos y con el fin de controlar si persistía la parasitemia se les practicó 7 xenodiagnósticos a cado uno con intérvalos de 8 hasta 278 días.

Se efectuó investigaciones epidemiológicas en el domicilio de un paciente que habiendo nacido en Lima, refirió haber residido en Cajamarca sólo 22 días.

Es importante mencionar- que ninguna de las personas consideradas en este estudio habían sido sometidas a pruebas diagnósticas para la enfermedad de Chagas con anterioridad. 


\section{RESULTADOS OBTENIDOS}

La reacción de fijación del complemento de Machado-Guerreiro fue positiva en 14 personas, entre las que hubo 4 hombres cuyas edades estaban entre 14 y 26 años; 6 entre 41 y 50; uno de 74 y además 3 mujeres: de 26,40 y 65 años. Tuvimos una reacción de fijación del complemento con resultado sospechoso y en otra el suero fue anticomplementario. Cuadro No 2 .

Del grupo de 14 casos con reacción de fijación del complemento de Machado-Guerreiro positiva, 6 habían nacido en Moquegua; 3 en Tacna; 3 en Arequipa; l en Cajamarca y l nacido en Lima y con residencia permanente en esta ciudad excepto por 22 días que estuvo on Celendín en compañía de su madre. Los dos tenian T. cruzi en sangre circulante y fue en ellos en los únicos en que se administró Primaquina a la dosis de 15 mgr. diarios durante 14 días consecutivos, comprobándose que éste medicamento no produjo eliminación de la parasitemia, pues siete xenodiagnósticos practicados a cada uno, tanto durante el tratamiento como después de éste, con intérvalos de 8 hasta 278 días, resultaron positivos. Cuadros No 3 y 4.

\section{Cuadro No 3. Casos de Enfermedod de Chagas diagnosticados entre 2) 4 residentes de Lima}

\begin{tabular}{cccc}
\hline $\begin{array}{c}\text { Lugar de nocimiento } \\
\text { O residencia anter. }\end{array}$ & $\begin{array}{c}\text { Método. } \\
\text { Fijación } \\
\text { complem. }\end{array}$ & $\begin{array}{r}\text { Diagnóstico usodo } \\
\text { Xenodiog. }\end{array}$ & $\begin{array}{c}\text { Total de } \\
\text { cosos }\end{array}$ \\
\hline Moquegua & 6 & 4 & 6 \\
Tacno & 3 & 1 & 3 \\
Arequipo & 3 & 1 & 2 \\
Cojomarco & 2 & & 3 \\
\hline
\end{tabular}

Se practicó 35 xenodiagnósticos a igual número de personas del grupo estudiado, entre los que estaban incluídos los 14 casos con reacción de Machado-Guerreiro positiva. En 6 de ellos se obtuvo resultado positivo. 
Es importante mencionar algunos hechos interesantes en relación con los paciertes que tenían la prueba del xenodiagnóstico positiva. Uno corresponde a una mujer de 40 años nacida en Tacna, pero que reside en Lima permanentemente desde hace 17 años, excepto por 22 días en 1957, que estuvo en Celendín. El segundo xenodiagnóstico positivo pertenece al hijo de la enferma arriba mencionada, quien como ya hemos referido tenía 14 años de edad, había nacido y residido permanentemente en Lima, con la excepción de los 22 días que estuvo con su madre en Celendín. Dos hermanos de 41 y 48 años que tienen xenodiagnóstico positivo han nacido en Mcquegua y residen en Lima desde hace 30 y 32 años

\section{Cuadro No 4. Comprabación de la parasitemia en 6 casos de Enfermedad de Chagas}

\begin{tabular}{|c|c|c|c|c|c|c|c|c|c|c|}
\hline$N^{\circ}$ & & 40 & años & 7 & Xenodiag. & en & 17 & meses. & Todos & positivos \\
\hline No & 2 & 15 & " & 7 & ", & $"$ & 17 & " & Todos & positivos \\
\hline No & 3 & 48 & " & 2 & " & $"$ & 12 & " & & positivos \\
\hline No & 4 & 41 & " & 1 & ॥ & & & & & positivo \\
\hline No & 5 & 45 & " & 1 & " & & & & & positivo \\
\hline Ne & 6 & 41 & " & 2 & $"$ & " & 12 & , & & positivos \\
\hline
\end{tabular}

respectivamente y en ambos éste examen ha demostrado la presencia de T. cruzi en Noviembre de 1961 y en Octubre de 1962. Otro de los pacientes con parasitemia tenía 11 años, también nacido en Moquegua, estudió instrucción primaria y media y una larga profesión en Lima, es decir, reside en esta ciudad desde 1930, con excepción de 15 días en 1952, 20 días en 1956 y 20 días en 1959 que regresó y residió en Moquegua. Afirma que en éstas tres oportunidades ya se había hecho campaña contra triatóminos en Moquegua y está seguro de no haber sido picado por chirimachas.

Queremos destacar el hecho que uno de nuestros casos, es un joven de 26 años que actualmente está en las Fuerzas Armadas, tiene sangre Rh negativa y por tal razón es muy solicitado para donar sangre.

Hemos tenido oportunidad de visitar y examinar las condiciones epidemiológicas del domicilio en Lima, del paciente de 14 años nacido en Lima; encontrando que la vivienda situada en un distrito popular, 
era pequeña, con tres habitaciones, piso de madera, paredes de adobe bastante bien revocadas con yeso y techos de madera, no existiendo grietas. Se verificó que en esta vivienda no había condiciones favorables para la reproducción de triatóminos y en la búsqueda minuciosa que realizamos con ayuda de linternas de mano no fue posible encontrar T. infestans.

\section{COMENTARIO}

Debido a que la población de Lima se ha incrementado en 232.4 $\%$ durante los últimos 21 años y gracias a la colaboración que en todo momento nos han brindado los colegas de la capital y especialmente los cardiólogos, nos fue relativamente fácil reunir un grupo de 214 personas residentes en Lima que habían nacido en zonas chagásicas o habían residido por periodos prolongados en ellas.

El diagnóstico de 14 casos de entermedad de Chagas en este grupo demuestra, por primera vez, que en Lima hay portadores de ésta Trypanosomiasis, 6 de los cuales tienen permanentemente T. cruzi en sangre circulante, pues repetidos Xenodiagnósticos practicados así lo demostraron. Estos hallazgos tienen importancia epidemiológica considerable, debido al peligro de diseminar la enfermedad mediante transfusiones sanguíneas (un paciente tiene Rh negativo). Teniendo en cuenta además, que en el grupo de enfermos que hemos diagnosticado hay dos mujeres jóvenes que pueden tener hijos, el peligro de diseminación es mayor aún, puesto que se ha probado la transmisión de ésta enfermedad por vía transplacentaria (5), o por la ingestión de líquido amniótico durante las maniobras del parto (6), y por la leche materna durante el periodo de lactancia (7).

Considerando que aún no hay tratamiento eficaz para la Trypanosomiasis Americana y estando demostrado que la infección puede persistir por más de veinte años (8), la presencia de T. cruzi en sangre periférica de personas residentes en Lima, constituye peligro constante, pues ellas pueden ser fuente de infección para triatóminos que fácilmente pueden ser traídos de otros regiones del Perú. Esto no es improbable, ya que así como se demostró que Triatomas habían sido llevados a Magdalena Vieja (1), y que tales insectos se habian instalado en una barriada popular de Lima (2); podría suceder cosa igual en otras zonas de la capital. La posibilidad mencionada es mayor si se considera la existencia de numerosas y fáciles vías de comunicación entre Lima y las zonas chagásicas del Perú. 
Estudios realizados por Tejada y Llanos, han demostrado que las características de la vivienda en determinadas zonas populares de la ciudad de Lima, reunen condiciones epidemiológicas que han permitido que Triatomas procedentes de otros lugates del Perú (2), se hubiesen establecido dos años antes de las investigaciones efectuadas por los autores mencionados. Creemos que infestaciones semejantes podrán repetirse en distritos populares que reunan tales condiciones epidemiológicas o en las nuevas barriadas marginales de Lima, que se constituyen con personas procedentes de diferentes lugares del país, inclusive zonas chagásicas, que al trasladarse a Lima traen no sólo sus enseres personales, sino también chirimachas, cuyes y otros animales domésticos que sirven de reservorio al T. cruzi.

\section{RESUMEN Y CONCLUSIONES}

Durante los últimos 30 meses hemos realizado investigaciones sobre la presencia de enfermedad de Chagas en residentes de la ciudad de Lima, que pueden resumirse de la manera siguiente:

1. Se ha demostrado la presencia de 14 casos de enfermedad de Chagas entre 214 residentes de la ciudad de Lima que habían nacido o residido por varios años en zonas chagásicas del Perú.

2. Se ha verificado que 6 de estas personas tienen T. cruzi en la sangre circulante varios años después de residir permanentemente en Lima, señalándose su importancia epidemiológica como posibles diseminadores de la enfermedad mediante transfusiones sanguíneas y como fuentes de infección para triatóminos.

3. El hallazgo de dos mujeres adultas jóvenes, entre los 14 casos diagnosticados, establece la posibilidad de disøminación de la enfermedad por vía transplacentaria y por iactancia materna.

4. La enfermedad de Chagas continúa extendiéndose en el torritorio nacional, siendo urgente poner en marcha una campaña de erradicación, exigir la práctica de la reacción de fijación del complemento de Machado-Guerreiro en los donadores de sangre y aplicar violeta de genciana en la proporción de 1/4,000 a la sangre conservada en bancos.

\section{SUMMARY}

Fourteen cases of Chages' disease were discovered by complementfixation tests among 214 residents of Lima who had come from en- 
demic areas of Perú. Xenodiagnostic tests conformed that six had Trypanosoma cruzi in the circulating blood many years after leaving an endemic area.

In view of the known establishment of the vector, Triatoma infestans, in certain districts of Lima and the great number of people who are leaving endemic areas to settle in the city, there in clearly indicated a danger of creating new endemic foci in Lima.

\section{AGRADECIMIENTOS}

Expresomos nuestro agradecimiento a los Doctores Amador Neghme, Julio Muñiz, L. Rodríguez C., Aurelio Peralto, C. Delgodo Cornejo, Felizo Knierim, Jeonnette Richords y a todos los médicos que han colaborado y dodo facilidodes pora la reolización de estas investigociones.

Agradecemos en formo especial o los Ayudantes señores Arquímedes Castilfo y Rolondo Gómez y al Técnico Sr. Victor Ourand J., por su eficiente colaboración en el laboratorio.

\section{B I BLIOGRAFIA}

1. Cornejo D., A.: Enfermedod de Chagas. Estado actual en el Perú. Proceedings of the Sixth Internotional Congresses On Tropical Medicine and Malaria. Lisboo, 3: 298-324, 1958.

2. Tejoda, A. y Llanos B.: Presencio del Triotomo infestons en lo ciudad de Lima. Rev. Med. Exp., Limo, 12: 90-98, 1958.

3. Bozicevich, J.; Hoyem, $H_{\text {. }}$, and Walston, V. M.: A method of conducting the 50 $\%$ Hemolysis end poind complement-fixation for parasitic diseases. Pub. Heoith Rep. 61: 529-534, 1946.

4. Knierim, F.: Técnica de la reocción de fijación del complemento según del $50 \%$ de hemólisis de Bozicevich aplicodo al diognóstico de lo enfermedod de Chagas. Bol. Chileno Parasit, 13 (4): 75-78, 1958.

5. Lisboa, Achilea C.: Sobre la formo congénita Da Doenca de Chagas. Estudo anátomo-potológico de 6 cosos. Rev. Inst. Med. Trop. Soo Poulo, 2 (6): 319 $334,1960$.

6. Notton-Lorrier, L.: Heredité des infections expérimentoles ó Sch. cruzi. Bull. Soc. Poth. Ex. 14 (4): 232-238, 1921.

7. Mazzo, S.; S. Montaño, A.; Benites, C. y Janzi, E. Z.: Transmisión del Schizotryponum cruzi, al niño por la leche de la madre con enfermedad de Chogas. M.E.P.R.A., pub., 28: $41-46,1936$.

8. Schlesinger, Poulo y Aaroo Burlomagui, B.: Coracteristicos clínicos de 75 casos de Cardiopatía Chagásica Crónico Observados. No Rio de Janeiro. Abstrocts del Sexto Congreso Internocionol de Cardiologío. Rio de Janeiro, 1960. 